Pour en lire plus : International Handbook of research on environmental education.

Yves Laberge

\author{
(2) OpenEdition \\ Journals \\ Édition électronique \\ URL : http://journals.openedition.org/ere/838 \\ DOI : $10.4000 /$ ere.838 \\ ISSN : 2561-2271 \\ Éditeur \\ Centr'ERE
}

Référence électronique

Yves Laberge, «Pour en lire plus : International Handbook of research on environmental education. », Éducation relative à l'environnement [En ligne], Volume 11 | 2014, mis en ligne le 20 décembre 2013, consulté le 24 septembre 2020. URL : http://journals.openedition.org/ere/838 ; DOI : https://doi.org/ $10.4000 /$ ere.838

Ce document a été généré automatiquement le 24 septembre 2020. 


\title{
Pour en lire plus : International Handbook of research on environmental education.
}

\author{
Yves Laberge
}

\section{RÉFÉRENCE}

Stevenson, R. B., Brody, M., Dillon, J., et Wals, A. (dir.) (2013.)International Handbook of research on environmental education. Washington DC, Abingdon et New York :

American Educational Research Association et Routledge, 2013, xii+576 p.

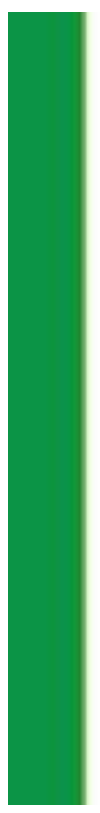

\section{Research on}

Environmental

Education

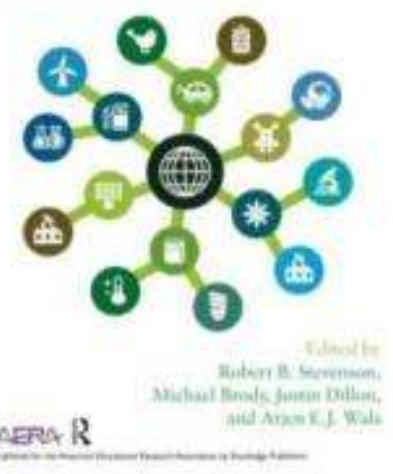

Publié conjointement par l'American Educational Research Association (AERA) et l'éditeur britanni-que Routledge, cet énorme manuel comprenant 51 chapitres fait l'état de la recherche en éducation 
relative à l'environnement, non seulement aux États-Unis, mais aussi ailleurs dans le monde et au Québec. Les auteurs proposent des avenues essentiellement conceptuelles et théoriques à propos d'une variété de dimensions, approches, tendances et sousthèmes, allant de l'étude des politiques environnementales jusqu'aux processus d'apprentissage, en incluant par ailleurs des avancées théoriques et méthodologiques. La plupart des chapitres présentent le point de vue d'une dizaine d'auteurs ayant réfléchi sur une question spécifique, en comparant ou en opposant leurs perspectives respectives. Ce n'est pas à proprement parler un livre pratique, mais plutôt une réflexion sur les manières de penser l'éducation relative à l'environnement.

1 Dans leur introduction, Robert B. Stevenson, Michael Brody, Justin Dillon et Arjen E.J. Wals reprennent diverses définitions de l'éducation relative à l'environnement formulées depuis les années 1970 afin de démontrer comment la discipline s'est progressivement transformée et élargie (p. 1). Dans le chapitre suivant, Annette Gough poursuit cet exercice de délimitation du domaine pour ensuite prôner un partage équitable des différentes conceptions du savoir, considéré comme étant "partiel, multiple, contradictoire », voire " potentiellement raciste » puisque les pollueurs visent parfois des zones où vivent des groupes ethniques défavorisés, que ce soit les Noirs des favélas brésiliennes, les townships d'Afrique du Sud ou encore les ghettos africainsaméricains des États-Unis (p.11). Plus loin, le cinquième chapitre met en évidence certaines tendances dans les thèses soutenues aux États-Unis dans le domaine de l'éducation relative à l'environnement au cours des trente dernières années, en indiquant les méthodes préconisées, notamment l'analyse de contenu (p. 45).

2 Les perspectives critiques abondent dans l'ensemble de ce livre. Par exemple au onzième chapitre, s'interrogeant sur le rôle du langage comme porteur de sens et d'une vision du monde, Lesley Le Grange soutient qu'il importe de cesser de concevoir l'éducation comme étant uniquement une transaction économique dans laquelle les parents d'élèves seraient perçus comme des acheteurs de services éducatifs (p. 109). Selon Lesley Le Grange, l'enga-gement critique des enseignants est essentiel en éducation à l'environnement, et la réflexion sur le langage devrait contribuer à un questionnement sur nos manières de vivre au quotidien et sur les dimensions les plus importantes à inclure dans les programmes éducatifs (p. 113). L'analyse du langage et $\mathrm{du}$ discours permettent d'appréhender un système de valeurs et les fondements idéologiques d'un discours.

Le Québec et les chercheurs québécois n'ont pas été négligés dans ce livre vraiment international avec la contribution rigoureuse de Tom Berryman et Lucie Sauvé qui poursuivent cette réflexion essentielle sur l'étude de la teneur du langage et des textes, prolongeant cette observation fondamentale de Jean Piaget voulant « que toute langue contient une logique et une cosmologie, et que l'enfant, apprenant à parler dans le même temps ou avant qu'il apprend à penser, pense en fonction du milieu social adulte " (Piaget, cité par Berryman et Sauvé, p. 143). Ce chapitre 14 de Tom Berryman et Lucie Sauvé approfondit substantiellement les propos plus vagues de Lesley Le Grange (au chapitre 11) qui basait son argumentation sur l'importance du langage sans toutefois mentionner les travaux fondateurs de Peter Berger et Thomas Luckmann sur la construction sociale de la réalité, et en citant seulement un ouvrage récent de Gert Biesta, et non le véritable initiateur de cette idée : Jean Piaget, dès 1926 (p. 108). Cette démonstration essentielle remontant aux origines était pourtant faite au chapitre 14 . 
En outre, certains chercheurs canadiens comme Bob Jickling et Paul Hart ont aussi contribué à ce livre.

Quelques chapitres explorent une question ou une dimension centrée sur l'éducation à l'environnement, par exemple le paysage, comparant les approches spécifiques de différents auteurs ayant précédemment conceptualisé ou théorisé le paysage (p. 281). Par ailleurs, le chapitre 37 sur la justice environnementale approfondit également le concept d'injustice environnementale puisque chaque concept devrait aussi être articulé en fonction de son contraire (p. 395).

5 Cette recension serait incomplète sans la mention des avancées méthodologiques suggérées dans plusieurs chapitres, entre autres dans le substantiel chapitre 41 où les enfants sont considérés comme des "chercheurs actifs", distinguant trois types apparemment similaires : la recherche sur les enfants, la recherche avec les enfants, et enfin la recherche faite par les enfants (p. 439). Bien qu'il soit difficile de synthétiser un ouvrage collectif de plus de cinq cents pages en seulement quelques lignes, les responsables de ce collectif (les professeurs Robert Stevenson, Michael Brody, Justin Dillon et Arjen Wals) réussissent ce périlleux exercice en mettant en évidence ce qui ressort de cet ensemble (chapitre 48). Dans la neuvième et dernière section, Stevenson, Brody, Dillon et Wals expliquent en termes réflexifs comment la discipline a changé au fil des ans en se concentrant sur des aspects devenus fondamentaux comme la vision du monde et les systèmes de croyances des élèves, des enseignants et des chercheurs en didactique («belief systems ») (p. 514). Parmi les autres intérêts de recherche émergents en éducation relative à l'environnement, on trouve également les dimensions culturelles, les espaces transculturels, ou encore l'analyse des discours sur l'environnement (p. 515).

6 Pour l'étudiant voulant entreprendre des études de maîtrise ou de doctorat, cet International Handbook of research on environmental education apparaitra comme une mine de renseignements et d'idées novatrices, un point de départ essentiel et utile pour la maitrise et le doctorat. Même les chercheurs aguerris y trouveront une multitude d'idées inspirantes et des perspectives novatrices. On comprend que les bibliothèques universitaires devront offrir ce livre étoffé à leurs usagers.

\section{AUTEUR}

\section{YVES LABERGE}

Professeur mi-temps, Faculté des arts, Université d'Ottawa 